\section{In the news}

\section{ROTAVIRUS VACCINES}

About half a million children under

five years of age die as a result of rotavirus infection each year. But hopes for a safe and effective vaccine have been raised by two recent studies published in The New England Journal of Medicine (5 January 2006).

The rotavirus vaccines Rotateq

(Merck \& Co.) and Rotarix

(GlaxoSmithKline) reduced the number of cases of severe

gastroenteritis in clinical trials by $98 \%$ and $85 \%$, respectively. Both vaccines also markedly reduced the number of infants requiring hospital treatment for symptoms of rotavirus infection (which include dehydration and diarrhoea).

Most deaths from rotavirus occur in developing countries, but almost all children worldwide are infected with rotavirus at least once by five years of age. And each year, 70,000 children in the United States receive hospital treatment (USA Today, 4 January 2006), as well as $\sim 1$ in 38 children in the United Kingdom by the age of five (BBC News, 5 January 2006).

Researchers have been attempting to develop a rotavirus vaccine since the 1970s. In 1998, RotaShield (Wyeth) was released on the market, but it was withdrawn within a year because a small number of individuals developed an intestinal blockage known as intussusception. Both of the new vaccines were therefore tested on a large number of infants

(in Europe, the United States and Latin America). Neither vaccine seemed to cause intussusception. "These are milestone results", says John Wecker, of PATH (New Scientist, 14 January 2006), a US non-profit organization that is working with Merck \& Co. and GlaxoSmithKline to set up trials in Asia and Africa.

Such trials are crucial, as Roger Glass and Umesh Parashar (Centers for Disease Control and Prevention, USA) write in The New England Journal of Medicine (5 January 2006): "Both vaccines will need to demonstrate their efficacy in the difficult settings of developing countries".

Davina Dadley-Moore

INFLAMMATION

\title{
T-bet links innate and adaptive immune responses
}

\author{
The transcription factor T-bet is \\ best known for its role in T helper 1 \\ $\left(\mathrm{T}_{\mathrm{H}} 1\right)$-cell differentiation, but now \\ new data indicate that T-bet expres- \\ sion by dendritic cells (DCs) is also \\ required for pro-inflammatory \\ cytokine production and T-cell \\ priming.
}

Because T-bet is highly expressed in tissues affected by rheumatoid arthritis in humans, Glimcher and colleagues set out to evaluate the role of T-bet in a mouse model of arthritis, collagen-antibody-induced arthritis (CAIA). In this model, disease is induced by administration

\section{INFLAMMATION}

\section{Switching on the inflammasome}

Assembly and activation of the inflammasome is an essential process in innate immune defence. The inflammasome is a cytosolic, multiprotein platform that allows activation of precursors of proinflammatory caspases, which then cleave the precursor of interleukin- $1 \beta$ (pro-IL-1 $\beta$ ) into the active form, the secretion of which leads to a potent inflammatory response. But little is known about the natural stimuli that lead to activation of the inflammasome and the mechanisms by which these stimuli accomplish this. Now, three studies published in Nature have identified bacterial components and endogenous 'danger' signals that activate inflammasomes that contain NALP3 (also known as cryopyrin).

The central component of an inflammasome is a member of the NALP family, and this protein associates with the adaptor protein apoptosis-associated speck-like these results

show that

NALP3 has a crucial role in host defence against certain bacteria and that it might be a proximal sensor of cellular stress and danger signals protein (ASC), which in turn recruits pro-inflammatory-caspase precursors (such as pro-caspase-1). NALP1, NALP2 and NALP3 have been shown to form inflammasomes, and the importance of NALP3 is well established, because mutations in the gene that encodes NALP3 (CIAS1) cause several autoinflammatory disorders. So, three groups of researchers set out to define the role of NALP 3 in inflammatory responses. Each group independently generated NALP3-deficient mice and then examined macrophages from these mice for their ability to process procaspase- 1 or pro-IL-1 $\beta$ and to secrete IL-1 $\beta$ in response to various microbial and non-microbial stimuli.

Núñez and colleagues examined macrophage responses to several Tolllike receptor (TLR) ligands and showed that NALP 3 is specifically required for pro-caspase- 1 cleavage and IL- $1 \beta$ production in response to the synthetic TLR7 ligands R837 (also known as imiquimod) and R848 (also known as resiquimod). These molecules are structurally similar to purine bases, so the authors also examined macrophage responses to bacterial RNA, which they speculated might be a natural ligand for NALP3, and they obtained similar results. Tschopp and colleagues similarly showed that crystals of monosodium urate (which 
of monoclonal antibodies specific for type II collagen together with lipopolysaccharide and is characterized by an early innate phase followed by a later adaptive phase. Compared with wild-type mice, the onset of CAIA in T-bet-deficient mice was delayed and was less severe in both the initial and the late phases.

Mice that are deficient in RAG2 (recombination-activating gene 2), which completely lack B and T cells, developed early joint inflammation, but the later phase was attenuated. And mice that are deficient in both T-bet and RAG2 were resistant to the induction of arthritis.

To further assess the cellular components of the innate phase, the authors carried out adoptivetransfer experiments. Transfer of wild-type DCs to T-bet-deficient mice on day 1 of CAIA induction restored inflammatory arthritis, and wild-type DCs restored the early phase response when transferred to mice that are deficient in both T-bet and RAG2. Consistent with this role for DCs in the early inflammatory phase, the authors showed that T-bet-deficient DCs produced less of the pro-inflammatory mediators interleukin- $1 \alpha$ and CC-chemokine ligand 3 (CCL3) and more CCL17, a ligand for the $\mathrm{T}_{\mathrm{H}}$ 2-cell-specific chemokine receptor CCR4, than wild-type DCs. Moreover, the ability of DCs to prime naive $\mathrm{T}$ cells in vivo was impaired in the absence of T-bet.

In light of these results, T-bet could be a target to control inflammation at multiple points in both innate and adaptive immune responses.

Lucy Bird

ORIGINAL RESEARCH PAPER Wang, J. et al. Transcription factor T-bet regulates inflammatory arthritis through its function in dendritic cells. J. Clin. Invest. 12 Jan 2006 (doi:10.1172/JCI26631)

\section{IN BRIEF}

\section{(O) T-CELL DEVELOPMENT}

Premature expression of chemokine receptor CCR9 impairs T cell development.

Uehara, S. et al. J. Immunol. 176, 75-84 (2006)

Only late $\mathrm{CD} 4^{-} \mathrm{CD} 8^{-}$double-negative (DN) thymocytes and $\mathrm{CD} 4^{+} \mathrm{CD}^{+}$double-positive thymocytes express $\mathrm{CC}$-chemokine receptor 9 (CCR9). To investigate whether expression of CCR9 controls the migration of thymocytes and their retention in the thymus, Uehara et al. generated transgenic mice in which CCR9 was expressed throughout T-cell development. $\mathrm{CD} 4^{+}$and $\mathrm{CD} 8^{+}$ single-positive (SP) thymocytes could exit the thymus, indicating that CCR9 downregulation is not essential for SP thymocyte emigration. By contrast, ectopic expression of CCR9 by early DN thymocytes partially blocked thymocyte development at the DN3 stage, and CD25 $5^{+}$thymocytes (DN2 and DN3 thymocytes) were found throughout the cortex rather than at the subcapsular region. These results indicate that the timing of CCR9 expression is important for normal thymocyte development and that this might be because CCR9 controls $\mathrm{CD} 25^{+}$thymocyte localization.

\section{HAEMATOPOIESIS}

Glycogen synthase kinase-3 is an in vivo regulator of hematopoietic stem cell repopulation.

Trowbridge, J. J. et al. Nature Med. 12, 89-98 (2006)

Glycogen synthase kinase 3 (GSK3) regulates several signalling pathways (such as the Notch and WNT signalling pathways) that affect the in vitro function of haematopoietic stem cells (HSCs). In this study, non-obese diabetic-severe combined immunodeficient mice were transplanted with HSCs from normal mice in the presence or absence of an ATP-competitive inhibitor of GSK3. Treatment with the GSK3 inhibitor increased recipient haematopoietic-cell reconstitution in both the short and the long term and thereby increased recipient survival. Further analysis showed that the GSK3 inhibitor increased the production of progenitor cells while maintaining the number of HSCs, leading the authors to suggest that treatment with GSK3 inhibitors might be a useful therapeutic approach to increase recipient haematopoietic-cell reconstitution after HSC transplantation.

are also structurally similar to purine bases and have recently been reported to be a danger signal) and calcium pyrophosphate dihydrate (both of which elicit inflammatory responses; known as gout and pseudogout, respectively) induce caspase-1-dependent IL-1 $\beta$ secretion by primed macrophages, and this relies on components of the inflammasome, including NALP3. Dixit and colleagues found that NALP3 is also required for pro-caspase- 1 activation by another danger signal, ATP (which, in vivo, is released into the extracellular environment by dying cells), and after exposure to certain Grampositive bacteria but not certain Gram-negative bacteria.

It is thought that, similar to recognition by TLRs, pathogen-associated molecular patterns interact with the leucinerich-repeat domains of NALPs, leading to activation of the inflammasome. provides eviden by the other two studies) that TLRs and NALP 3 induce immune responses through recognition of the same or similar microbial structures and that IL- $1 \beta$ secretion is independently regulated at two stages: the first is TLR dependent and results in nuclearfactor- $\mathrm{KB}$-mediated transcription of the gene that encodes pro-IL-1 $\beta$, and the second is NALP 3 dependent and results in cleavage of pro-IL-1 $\beta$ into IL- $1 \beta$.

Taken together, these results show that NALP3 has a crucial role in host defence against certain bacteria and that it might be a proximal sensor of cellular stress and danger signals. In addition, these studies provide insight into the mechanisms of autoinflammatory disorders that involve NALP3.

Davina Dadley-Moore

\section{ORIGINAL RESEARCH PAPERS}

Kanneganti, T.-D. et al. Bacterial RNA and small antiviral compounds activate caspase-1 through cryopyrin/Nalp3. Nature 11 Jan 2006 (doi:10.1038/nature04517)| Martinon, F.,

Pétrilli, V., Mayor, A., Tardivel, A. \& Tschopp, J. Gout-associated uric acid crystals activate the NALP3 inflammasome. Nature 11 Jan 2006 (doi:10.1038/nature04516)| Mariathasan, S. et al. Cryopyrin activates the inflammasome in response to toxins and ATP. Nature 11 Jan 2006 (doi:10.1038/nature04515)

\section{HAEMATOPOIESIS}

Antagonistic effect of CCAAT enhancer-binding protein- $\alpha$ and Pax 5 in myeloid or lymphoid lineage choice in common lymphoid progenitors.

Hsu, C.-L. et al. Proc. Natl Acad. Sci. USA 103, 672-677 (2006) Although lymphoid-lineage-committed progenitors, such as common lymphoid progenitors (CLPs) and pro-T cells, are mainly destined to become lymphocytes, they maintain a latent myeloid differentiation potential, which can be initiated by stimulation through ectopic interleukin-2 receptor (IL-2R). This study shows that IL-2R triggering of CLPs and pro-T cells results in rapid upregulation of the myeloid-related transcription factor CCAAT enhancer-binding protein- $\alpha$ (C/EBP $\alpha)$. Moreover, ectopic expression of $C / E B P \alpha$ in these cells leads to myeloidcell development. $\mathrm{C} / \mathrm{EBP} \alpha$ expression completely suppressed expression of the B-cell-lineage transcription factor PAX5 in CLPs and pro-T cells. By contrast, IL-2R stimulation of pro$B$ cells did not lead to lineage conversion, owing to insufficient downregulation of PAX 5 expression by $\mathrm{C} / \mathrm{EBP} \alpha$, indicating that complete loss of PAX5 is crucial for lymphoid-to-myeloid-lineage conversion. 